UCRL-JC-127710

\title{
PREPRINT
}

\section{Ab Initio Calculation of Tight-Binding Parameters}

\author{
A.K. McMahan
}

J.E. Klepeis

This paper was prepared for submittal to the 1997 Materials Research Society Fall Meeting

Boston, $M A$

December 1-5, 1997

December 1997

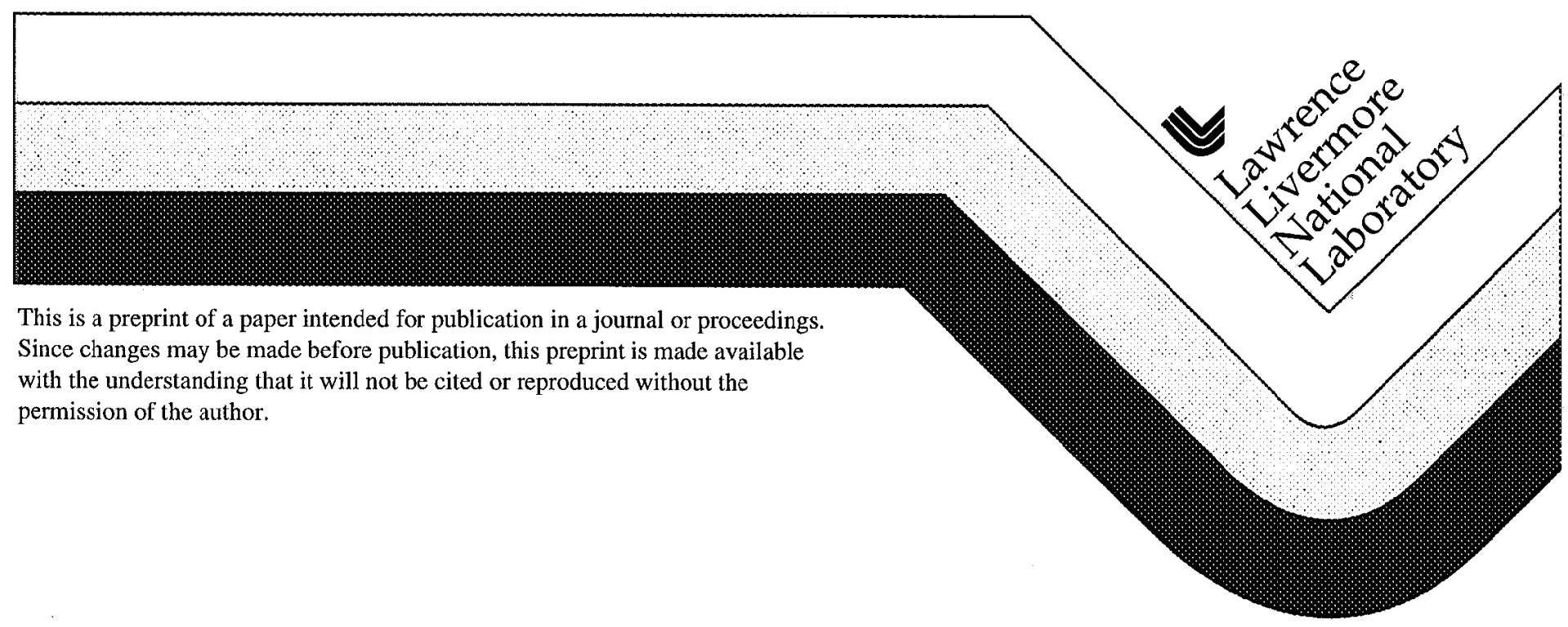




\section{DISCLAIMER}

This document was prepared as an account of work sponsored by an agency of the United States Govermment. Neither the Linited States Government nor the University of Califomia nor any of their employees, makes any warranty, express or implied, or assumes any legal liability or responsibility for the accuracy, completeness, or usefulness of any information, apparatus, product, or process disclosed, or represents that its use would not intinge privately owned rights Reference herein to any specific commercial product, process, or service by trade name, trademark, manufacturer, or othemise does not necessarily constitute or imply its endorsement, recommendation, or favoring by the Linited States Government or the Liniversity of Califomia. The views and opinions of authors expressed herein do not nesessarily state or ret!ec those of the Lnited States Government or the Liniversity of Califomia and stall not be used for acierssing or product endorsement purposes. 


\section{AB INITIO CALCULATION OF TIGHT-BINDING PARAMETERS}

A. K. MCMAHAN, J. E. KLEPEIS

Lawrence Livermore National Laboratory, P.O. Box 808, Livermore, CA 94551

\section{ABSTRACT}

We calculate $a b$ initio values of tight-binding parameters for the $f$-electron metal Ce and various phases of $\mathrm{Si}$, from local-density functional one-electron Hamiltonian and overlap matrix elements. Our approach allows us to unambiguously test the validity of the common minimal basis and two-center approximations as well as to determine the degree of transferability of both nonorthogonal and orthogonal hopping parameters in the cases considered.

\section{INTRODUCTION}

The success of local-density functional calculated total energies and forces and the synthesis of these techniques with molecular dynamics has opened up major new possibilities for atomistic simulation $[1,2]$. The expense of such ab initio molecular dynamics calculations, however, remains a drawback which has focused attention on the use of cheaper electronic calculations as in tight-binding molecular dynamics [3]. The tight-binding totalenergy representations required for this application are typically developed by fitting large $a b$ initio generated data bases of band structures, total energies, and possibly forces over the range of stoichiometry, coordination, and atomic volume which are of interest [4]- [10]. Unfortunately the nonlinear optimization required in such fits becomes more and more difficult as the number of chemical species increases, and the time required to generate the data bases can also become prohibitive. Ideally one would like to automate the process, perhaps with $a b$ initio calculation of the representation itself. While this is a challenging goal, it may be useful to begin with an examination of what our local-density functional methods are actually doing from a tight-binding perspective. This approach enables direct tests of the accuracy and transferability of the resulting tight-binding parameters while at the same time providing insight into the general characteristics required for a robust parametrization.

In a recent paper [11], we have shown that tight-binding parameters may be directly and simply calculated from the $\mathbf{k}$-dependent one-electron Hamiltonian and overlap matrix elements generated by any band structure method which is formulated in or can be projected onto a localized basis. After briefly reviewing these theoretical concepts and the FP-LMTO method [12] which we use to calculate these matrix elements, we extend such calculations here to include an $f$-electron metal, Ce. By a comparison of the ab initio bands and those reconstructed from the calculated tight-binding parameters we assess two major approximations customary in tight-binding: use of a minimal basis and the two-center expansion. We then report ncw results for a range of coordinations in $\mathrm{Si}$, and discuss the issue of transferability in terms of the $a b$ initio calculated nonorthogonal parameters. Finally we turn to the impact of orthogonalization on transferability, again for the case of $\mathrm{Si}$, and then provide a summary. 


\section{THEORETICAL METHODS}

\section{Tight-binding Parameters}

To facilitate subsequent discussion, it is useful to briefly review the formal relations between matrix elements of the one-electron Hamiltonian, $H$, or overlap, $O$, operators and Slater-Koster tight binding parameters. For simplicity, we consider only one atom per primitive cell, as more general expressions are given elsewhere [11]. In particular, let $|\mathbf{R} \ell m\rangle$ designate an orbital of angular and magnetic quantum numbers $\ell$ and $m$, respectively, at the lattice site $\mathbf{R}$. Direct and k-space matrix elements of $H$ are related as usual by

$$
\left\langle 0 \ell m|H| \mathbf{R} \ell^{\prime} m^{\prime}\right\rangle=\frac{1}{N} \sum_{\mathbf{k}} e^{-i \mathbf{k} \cdot \mathbf{R}} H_{\ell m, \ell^{\prime} m^{\prime}}^{\mathrm{k}}
$$

where the former are real as we consider $m$ to represent the customary real linear combinations of orbitals for each $\ell[13,14]$.

The intersite $(R \neq 0)$ matrix elements may be approximated by a two-center expansion in terms of Slater-Koster hopping parameters, $t_{\ell \ell^{\prime} \mu}(R)$.

$$
\left\langle 0 \ell m|H| \mathbf{R} \ell^{\prime} m^{\prime}\right\rangle \approx \sum_{\mu} g_{\mu}\left(\ell m, \ell^{\prime} m^{\prime}, \hat{\mathbf{R}}\right) t_{\ell \ell^{\prime} \mu}(R)
$$

Here, $g_{\mu}\left(\ell m, \ell^{\prime} m^{\prime}, \hat{\mathbf{R}}\right)$ are (real) geometric factors $[13,14], \mu$ is the magnetic quantum number about the bond axis $\mathbf{R}, \mu=\sigma, \pi, \cdots, \min \left(\ell, \ell^{\prime}\right)$, and $R \equiv|\mathbf{R}|, \hat{\mathbf{R}} \equiv \mathbf{R} / R$. Orthogonality relations [11] among the $g_{\mu}$ give

$$
t_{\ell \ell^{\prime} \mu}(R)=\left(2-\delta_{\mu \sigma}\right)^{-1} \sum_{m, m^{\prime}} g_{\mu}\left(\ell m, \ell^{\prime} m^{\prime}, \hat{\mathbf{R}}\right)\left\langle 0 \ell m|H| \mathbf{R} \ell^{\prime} m^{\prime}\right\rangle
$$

which in conjunction with Eq.(1), uniquely defines the Slater-Koster hopping parameters in terms of the $\mathrm{k}$-dependent one-electron Hamiltonian matrix elements. Hopping parameters, $s_{\ell \ell^{\prime} \mu}(R)$, are similarly defined from the overlap matrix elements.

The essential nature of the two-center approximation in Eq.(2) is the use of the SlaterKoster geometric functions, $g_{\mu}\left(\ell m, \ell^{\prime} m^{\prime}, \hat{\mathbf{R}}\right)$, as a matrix basis for the $m, m^{\prime}$-dependence of the $\left\langle 0 \ell m|H| \mathbf{R} \ell^{\prime} m^{\prime}\right\rangle$. Equation (2) is approximate since this basis is in general incomplete. The $t_{\ell \ell^{\prime} \mu}$ are nonetheless precisely defined as the projections of $\left\langle 0 \ell m|H| \mathbf{R} \ell^{\prime} m^{\prime}\right\rangle$ onto the existing matrix basis functions. Three-center contributions to the matrix elcments arising from one-electron potential terms in $H$ close to the bond axis, $\mathbf{R}$, are likely also to have significant projections onto these basis functions. "Non-two-center" effects is therefore perhaps a better description of the inadequacies of Eq.(2) than is three-center terms. Conversely, the extent to which three-center terms are absorbed into the effective $t_{\ell \ell^{\prime} \mu}$ is a source of non transferability as will be discussed subsequently.

The intrasite matrix elements, $\left\langle 0 \ell m|H| 0 \ell^{\prime} m^{\prime}\right\rangle$, define site energy, $\varepsilon_{\ell}$, and Slater-Koster crystal field parameters, $\chi_{\ell \ell^{\prime} \mu}(R)$.

$$
\left\langle 0 \ell m|H| 0 \ell^{\prime} m^{\prime}\right\rangle \approx \varepsilon_{\ell} \delta_{\ell \ell^{\prime}} \delta_{m m^{\prime}}+\sum_{\mathbf{R}, \mu}^{R \neq 0} g_{\mu}\left(\ell m, \ell^{\prime} m^{\prime}, \hat{\mathbf{R}}\right) \chi_{\ell \ell^{\prime} \mu}(R)
$$

The latter arise from two-center expansion of one-electron potential terms in $H$ at sites $\mathbf{R}$. In contrast to the hopping parameters, the $\varepsilon_{\ell}$ and $\chi_{\ell \ell^{\prime} \mu}(R)$ are underdetermined by the 
matrix elements of a single structure. The intrasite matrix elements behave as if they were characterized by generally a small number of effective site energies, $\varepsilon_{\ell}^{\text {eff }}$, and crystal field interactions, $\chi_{\ell \ell^{\prime} \mu}^{\mathrm{eff}}(R)$, which can be determined. The former are given by

$$
\begin{aligned}
\varepsilon_{\ell}^{e f f} & =\frac{1}{2 \ell+1} \sum_{m}\langle 0 \ell m|H| 0 \ell m\rangle, \\
& =\varepsilon_{\ell}+\frac{1}{2 \ell+1} \sum_{\mathbf{R}, \mu}^{R \neq 0}\left(2-\delta_{\mu \sigma}\right) \chi_{\ell \ell \mu}(R),
\end{aligned}
$$

showing that site energies extracted from either one-electron matrix elements or band structure are intrinsically dependent on local environment. The similarity of Eq.(6) to a pair potential sum, moreover, provides justification [11] for using environmentally dependent site energies in place of interatomic potentials in tight-binding total-energy representations [7]. The remaining effective crystal field parameters are discussed elsewhere [11].

\section{EP-LMTO Calculations}

We use the FP-LMTO method [12] to obtain the minimal basis matrix elements from which the tight binding parameters are calculated according to the above expressions. Details are given elsewhere [11]. In brief, the FP-LMTO method is first used in its most rigorous multiple- $\kappa$ form to obtain self-consistent one electron potentials and accurate band structures for test comparisons. A linked or contracted minimal basis is then chosen by minimization of the occupied one-electron eigenvalue sum using the fixed self-consistent potentials. The variables optimized in this way include the relative coefficients of the two augmented spherical Hankel functions which form each minimal basis function. The decay energies, $-\kappa^{2}$, of the Hankel function tails correspond to $\kappa_{1}^{2}=0.5$ Ry with $\kappa_{2}^{2}$ also optimized in the range 1-1.5 Ry. The tight binding parameters are then calculated from the resultant minimal basis Hamiltonian and overlap matrices. When discussing issues of transferability between different structures, as here for Si, we also first perform a unitary transformation which "rotates" all Hamiltonian and overlap matrices so as to guarantee a perfectly transferable, two-center overlap while preserving the band structure. This allows all issues of non-two-center effects and transferability to be addressed solely in terms of the Hamiltonian hopping parameters. All muffin-tin radii in both multiple- $\kappa$ and minimal basis calculations were taken to be nearly (reduced by $\sim 1 \%$ ) touching, unless otherwise stated.

\section{APPROXIMATIONS}

Tight binding representations of the one electron Hamiltonian typically make two approximations: (1) use of a minimal basis, and (2) the two-center approximation. We examine these approximations separately here for the case of fcc Ce at the experimental volume of the $\alpha$ phase.

Figure 1(a) compares our best multiple- $\kappa$ band structure (solid lines) to the linked minimal basis representation (dots). The former calculations included three $\left(\kappa^{2}=0.01,1\right.$, $2.3 \mathrm{Ky}$ ) augmented spherical Hankel basis functions of each $\ell m$ type for $s$ and $p$ states, two $\left(\kappa^{2}=0.01,1 \mathrm{Ry}\right)$ for $d$ states, and one $\left(\kappa^{2}=0.01 \mathrm{Ry}\right)$ for $f$ states. The dimension of the Hamiltonian and overlap matrices was therefore $29=3 \times 1+3 \times 3+2 \times 5+1 \times$ 7 at each $\mathbf{k}$ point. Our linked minimal basis treatment used two augmented spherical 

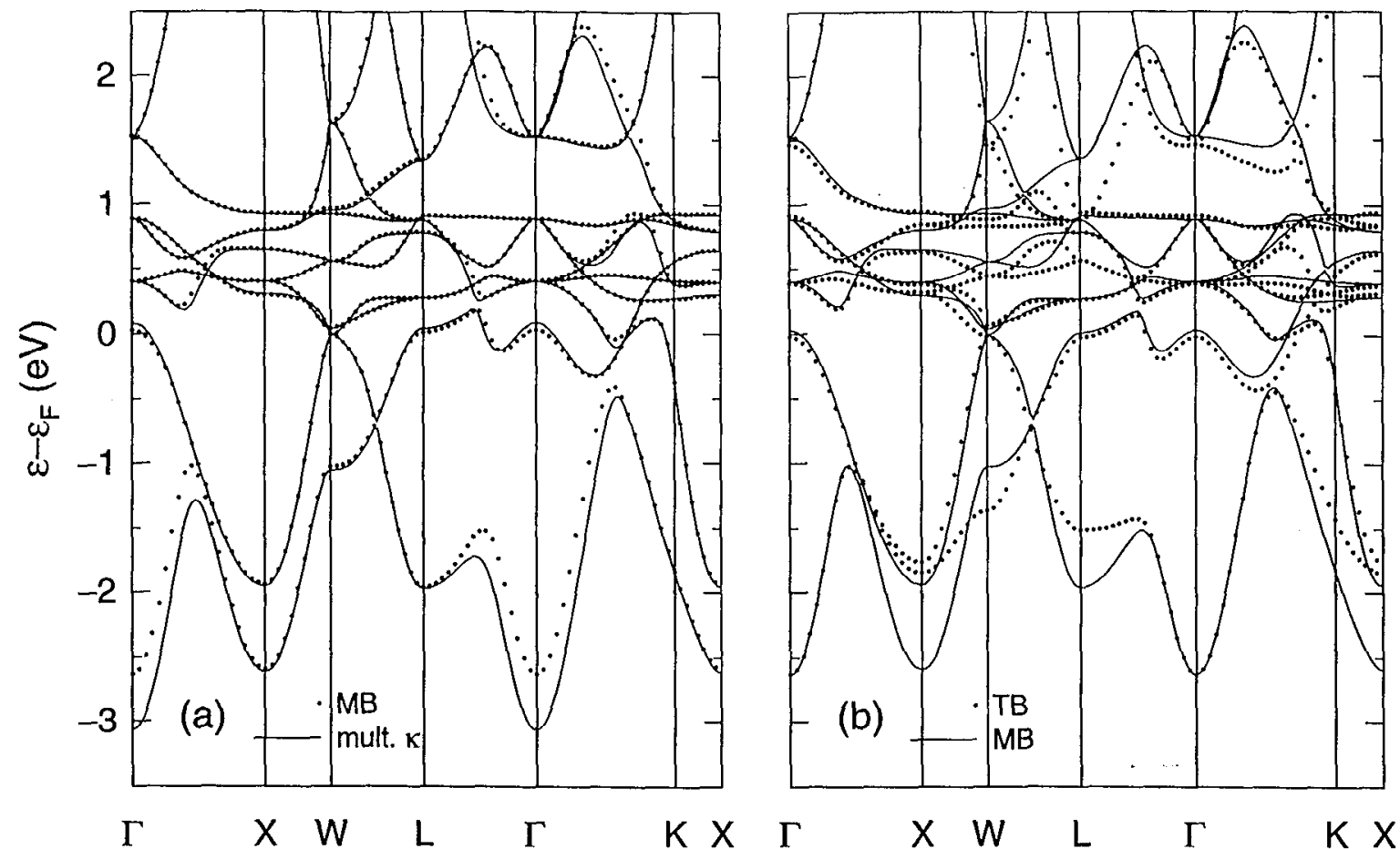

Figure 1: Band structure for fcc Ce: (a) comparison of our best multiple- $\kappa$ bands (lines) to the results from the minimal basis (dots), and (b) comparison of the minimal basis bands (lines) to the tight-binding bands reconstructed from the tight-binding parameters calculated from the minimal basis matrix elements (dots).

Hankel functions $\left(\kappa^{2}=0.5,1.2 \mathrm{Ry}\right)$ to represent each atomic orbital; however, the relative coefficients were fixed so that the linear combination for each $\ell m$ served as a single atomic orbital, leading to Hamiltonian and overlap matrices of dimension 16. The important distinction between multiple- $\kappa$ and linked calculations is that the relative coefficients of different augmented spherical Hankel functions corresponding to the same $\ell m$ may vary with $\mathbf{k}$-vector for different eigenfunctions in the first case, whereas they are fixed and kindependent in the latter. These coefficients come out of solving the one-electron eigenvalue problem in the first case, while they were obtained by optimization of the occupied oneelectron eigenvalue sum, as noted above, in the latter case.

The agreement between the two sets of bands in Fig. 1(a) is on the whole quite good. The rms differences are 0.17 and $0.02 \mathrm{cV}$ for the two lowest bands, and $0.02 \pm 0.01 \mathrm{eV}$ for the seven predominantly $f$ bands between $0.2-1 \mathrm{eV}$, as determined at 145 points throughout the Brillouin zone. The only significant discrepancy is in the lowest band, primarily at and nearby the pure-s $\Gamma$ point. We believe this is due to the minimal basis orbital being too localized, given analogous behavior for other materials where we have sometimes found unusually slow convergence at $s$-like $\Gamma$ points as a function of shell cut-off in our $a b$ initio tight-binding representations. Moreover, since $s-s$ contributions from different directions and shells should contribute constructively at the $\mathrm{I}^{\prime}$ point, a symptom of a too-localized $s$ basis orbital should be a too-narrow $s$ band with a bottom which is too high in energy as is seen. While we would expect smaller values of $\kappa^{2}$ to remedy this problem in the minimal basis treatment, this would also lead to longer ranged tight-binding representations.

Figure 1(b) compares the minimal basis band structure for fcc Ce (solid lines) to the 
tight binding bands (dots) reconstructed using hopping parameters given by Eq.(3) and its overlap analog, the effective site energies given by Eq.(5), and four effective nearneighbor crystal field interactions (one $p f$, one $d d$, and two $f f$ ) obtained as discussed in [11]. The cut-off for the tight binding parameters was $d_{\max } / a=2.25$, where $a$ is the lattice constant. We verified convergence with $d_{\max }$ by obtaining essentially identical bands using $d_{\text {max }} / a=2.99$.

The rms differences between the two lowest bands in Fig. 1(b) are 0.24 and $0.10 \mathrm{eV}$, respectively, and $0.08 \pm 0.04 \mathrm{eV}$ for the seven predominantly $f$ bands. The most serious discrepancies occur for the lowest band, at and near the mostly $d$-like $X$ (sd hybrid), $W$ $(p d f)$, and $L$ points $(s d)$. All differences between the two sets of bands in Fig. 1(b) are due entirely to non-two-center effects, i.e., to $m, m^{\prime}$ dependence of the matrix elements in Eq.(1) which can not be spanned by the two-center geometric functions. Since such dependence is likely to arise from three-center contributions to the matrix elements, and these should become less important for more localized bases, one would expect better agreement between the minimal basis and tight-binding bands for more localized bases. We find this to be the case in calculations where we have taken the minimal basis orbitals to be just the $\kappa^{2}=0.5$ Ry augmented spherical Hankel functions. The near-neighbor overlap hopping parameters for this basis are smaller by 24,47 , and $\sim 70 \%$ for the $s s \sigma, s d \sigma$, and $d d \mu$ parameters, respectively, compared to the linked basis. The corresponding rms agreement for the lowest band between minimal basis and tight binding results is improved for this basis by a factor of 4, to $0.06 \mathrm{eV}$, over the result using the linked minimum basis. Unfortunately, while there is improved agreement between the minimal basis and tight binding bands, both are in poor agreement with our rigorous multiple- $\kappa$ bands.

The rms agreement between minimal basis and tight binding $4 f$ bands in Fig. 1(b) is about $10 \%$ of the $4 f$ band width. One might have thought that the $4 f$ orbitals were already sufficiently localized even in the linked basis to be immune from non-two-center effects. Note, however, that the number of two-center geometric matrices goes up only as $\ell$ while the size of the $m, m^{\prime}$ matrices which they are asked to represent increases by $(2 \ell+1)^{2}$. It would appear that non-two-center effects become worse with increasing $\ell$.

\section{TRANSFERABILITY}

In previous $a b$ initio calculations of the hopping parameters for the silicon-boron system, we obtained relatively transferable parameters in so far as chemical and atomic-volume variation were concerned, however, there were indications of significant coordination dependence in the parameters [11]. We cxplore the coordination issue further here for the face centered cubic (fcc), body centered cubic (bcc), simple cubic (sc), and cubic diamond (cd) phases of $\mathrm{Si}$, i.e., coordinations of $12,8,6$, and 4 , respectively.

Figure 2 shows the ratios $-t_{\ell \ell^{\prime} \mu}(d) / s_{\ell \ell^{\prime} \mu}(d)$ for the $s p$ subset of our calculated parameters for the four phases of $\mathrm{Si}$. The calculated values are shown as the data points which appear in triples for each neighbor distance, corresponding to the theoretical equilibrium volume, $V_{0}$, and $(1 \pm 0.2) V_{0}$. The lines connecting these points pass through the first neighbors for all three volumes, then the second, and so on. These parameters correspond to the rotated matrices described above, so that the $s_{\ell \ell^{\prime} \mu}$ are perfectly transferable by construction. A rigid shift of any band structure by $\Delta \varepsilon$ will change the corresponding $t_{\ell \ell^{\prime} \mu} \rightarrow t_{\ell \ell^{\prime} \mu}+\Delta \varepsilon s_{\ell \ell^{\prime} \mu}$ in the case of a nonorthogonal basis. We have therefore plotted the $-t_{\ell \ell^{\prime} \mu} / s_{\ell \ell^{\prime} \mu}$ ratios in Fig. 2 on the same energy scale, so that such a rigid band shift for a given structure may 


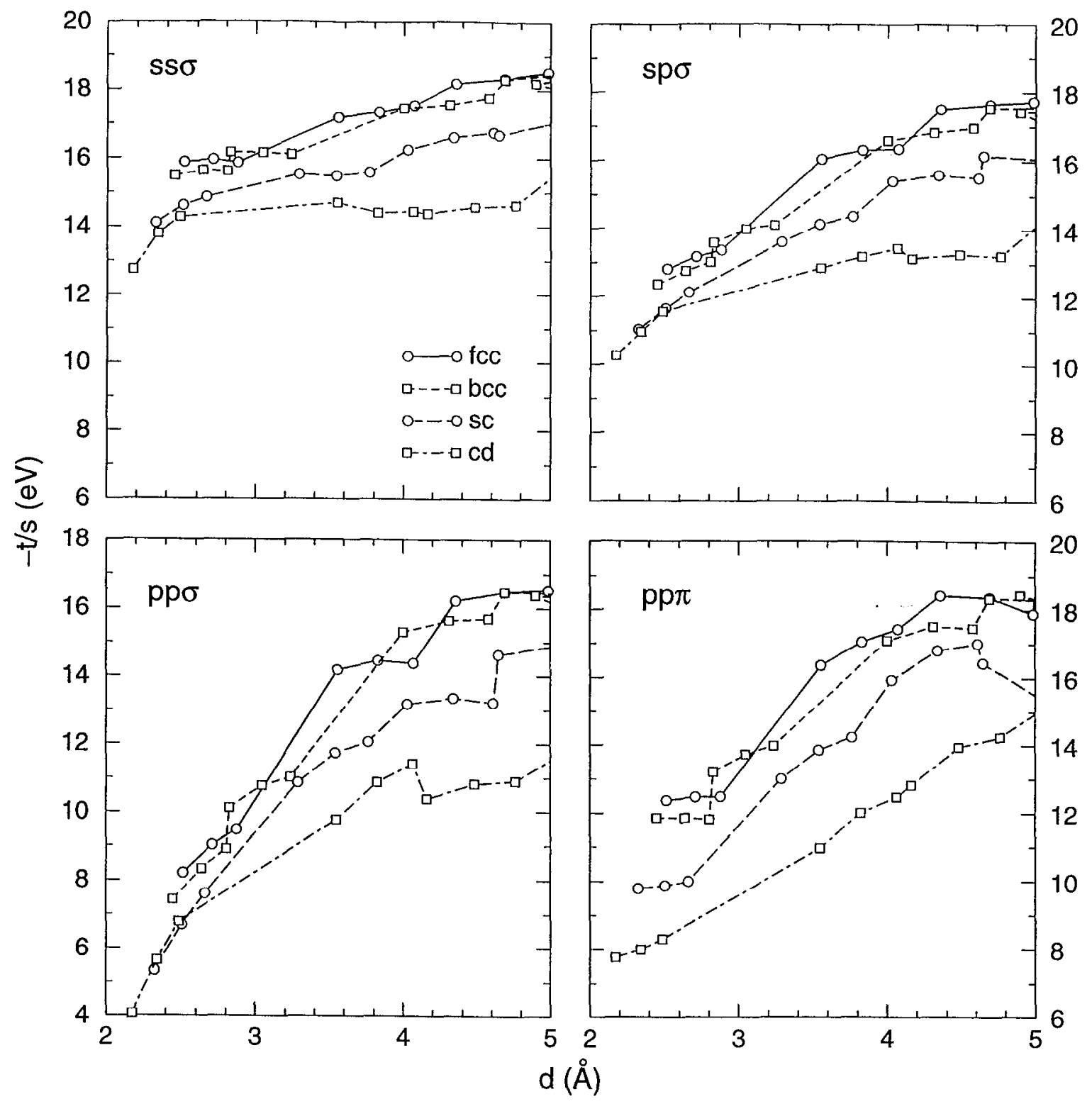

Figure 2: Negative ratios of the Hamiltonian and overlap hopping parameters, $-t_{\ell \ell^{\prime} \mu} / s_{\ell \ell^{\prime} \mu}$, for Si structures.

be envisioned by moving all four $\ell \ell^{\prime} \mu$ curves for that structure up or down by the same amount. No such shifts have been performed; however, the results in Fig. 2 are as obtained directly from the minimal basis matrix elements. The full set of calculated $s p d$ parameters reproduce our best multiple- $\kappa$ band structures to within an rms of $0.1-0.2 \mathrm{eV}$ for states up to $4 \mathrm{eV}$ above the Fermi level, an energy range of 15-20 eV. Note that the actual magnitudes of the $t$ 's change by factors of $\sim 20$ or more over the $d$ range covered in Fig. 2, and that one could draw smooth curves through the calculated $t_{\ell \ell^{\prime} \mu}(d)$ for all volumes and neighbors of a given structure which would follow this variation to within about $5 \%$ or so.

The essential conclusions to be drawn from Fig. 2 are that the high coordination fcc and bcc parameters track each other fairly well while the lower coordination structures have systematically smaller $t$ 's. Even if rigid band shifts were used to align the near-neighbor parameters more closely for all structures, the latter comment would still apply to the 2nd 
and 3rd neighbor values with spreads of $10-20 \%$ and $15-35 \%$, respectively [15]. In the remainder of this section, we discuss these conclusions to see if they are consistent with intuitive expectations.

Suppose that the self-consistent one-electron Hanilionian can be approximated by

$$
H=\frac{-\hbar^{2}}{2 m} \nabla^{2}+\sum_{\mathbf{R}} v_{\mathbf{R}},
$$

where the $v_{\mathbf{R}}(\mathbf{r})$ are spherically symmetric about $\mathbf{R}$ and

$$
\left(\frac{-\hbar^{2}}{2 m} \nabla^{2}+v_{\mathbf{R}}\right)|\mathbf{R} \ell m\rangle=\varepsilon_{\ell}|\mathbf{R} \ell m\rangle
$$

It then follows from Eq.(3) and the $g_{\mu}$ orthogonality relations that

$$
\begin{aligned}
t_{\ell \ell^{\prime} \mu}(R)= & \frac{1}{2}\left(\varepsilon_{\ell}+\varepsilon_{\ell^{\prime}}\right) s_{\ell \ell^{\prime} \mu}(R)+\Delta t_{\ell \ell^{\prime} \mu}(R) \\
& +\left(2-\delta_{\mu \sigma}\right)^{-1} \sum_{\mathbf{R}^{\prime}}^{\neq 0, \mathbf{R}} \sum_{m, m^{\prime}} g_{\mu}\left(\ell m, \ell^{\prime} m^{\prime}, \hat{\mathbf{R}}\right)\left\langle 0 \ell m\left|v_{\mathbf{R}^{\prime}}\right| \mathbf{R} \ell^{\prime} m^{\prime}\right\rangle
\end{aligned}
$$

where $\Delta t_{\ell \ell^{\prime} \mu}(R)$ comes from the two-center expansion of $\left\langle 0 \ell m\left|\frac{1}{2}\left(v_{0}+v_{\mathbf{R}}\right)\right| \mathbf{R} \ell^{\prime} m^{\prime}\right\rangle$. While the $v_{R}$ may of course be structure dependent, a more obvious source of environmental dependence of the $t_{\ell \ell^{\prime} \mu}$ will come from the three-center matrix elements in the last term of Eq.(9). This expression is particularly simple for the $s s \sigma$ case where $g_{\sigma}(s, s, \hat{\mathbf{R}})=1$. Since one might expect the $v_{\mathbf{R}}$ to be negative, these three-center contributions to $t_{s s \sigma}$ should then all make positive contributions to the $-t_{s s \sigma} / s_{s s \sigma}$ ratios plotted in Fig. 2 . An examination of the fcc, bcc, cd, and sc structures shows that the closer packed phases tend to have more atoms closer to the shorter bond axes $\mathbf{R}$ than do the lower coordination structures, which is consistent at least on geometric grounds with the $-t_{s s \sigma} / s_{s s \sigma}$ ordering in Fig. 2.

Additional insight is provided by the canonical band theory of Andersen and coworkers [16]. For pure $\ell$-bands, one may write the second-order one-electron eigenvalue problem in the form

$$
\left[C_{\ell}+\left(\Delta_{\ell}-\gamma_{\ell} C_{\ell}\right) \mathbf{S}_{\ell \ell}\right] x=\lambda\left(1-\gamma_{\ell} \mathbf{S}_{\ell \ell}\right) x,
$$

where $\lambda$ is the eigenvalue and $\mathbf{S}_{\ell \ell}=\left[S_{\mathbf{R} \ell m, \mathbf{R}^{\prime} \ell m^{\prime}}\right]$ is the bare canonical structure constant matrix. Material dependent potential parameters are the band center, $C_{\ell}$, the band width or inverse mass, $\Delta_{\ell}$, and a band distortion or nonorthogonality parameter, $\gamma_{\ell}$. Given the definition of the canonical structure constants [16], it can be shown that Eq. (10) is formally equivalent to the nonorthogonal tight-binding problem

$$
\begin{aligned}
\varepsilon_{\ell} & =C_{\ell} \\
s_{\ell \ell \mu}(d) & =\gamma_{\ell} f_{\ell \ell \mu} 2(2 \ell+1)\left(R_{\mathrm{WS}} / d\right)^{2 \ell+1} \\
t_{\ell \ell \mu}(d)-\varepsilon_{\ell} s_{\ell \ell \mu}(d) & =-\Delta_{\ell} f_{\ell \ell \mu} 2(2 \ell+1)\left(R_{\mathrm{WS}} / d\right)^{2 \ell+1}
\end{aligned}
$$

where $R_{\mathrm{WS}}$ is the Wigner-Seitz radius, $f_{s s \sigma}=1, f_{p p(\sigma, \pi)}=-2,1$, and $f_{d d(\sigma, \pi, \delta)}=6,-4,1$.

One of the strengths of the LMTO-ASA method [16] has been its separation of material dependence (potential parameters) from structure dependence (structure constants). The tight-binding representation given by Eqs. (11-13) is explicitly transferable to the extent that the $C_{\ell}, \gamma_{\ell}$, and $\Delta_{\ell}$ are independent of structure, which is known to be the case to a high 
degree for the relatively close packed structures for which the LMTO-ASA method is most accurate. Moreover, there has been considerable success in the calculation of structural energy differences for relatively close packed materials using canonical band theory [17] as well as by the LMTO force-relation technique [18] which both embody these same ideas of using fixed potential parameters which we equate to transferable representations. All of this experience, as well as the success of new tight-binding total energy representations for the transition metals [7], suggest a rather high degree of transferability in the close packed limit, in agreement with Fig. 2.

\section{ORTHOGONAL BASES}

Here we consider the suitability of orthogonal bases by performing Löwdin transformations [19], $\mathbf{H}^{\text {or }}=\mathrm{O}^{-1 / 2} \mathbf{H O}^{-1 / 2}$, to create (k-dependent) Hamiltonian matrix elements, $\mathbf{H}^{\text {or }}$, in an orthogonal basis. Unfortunately, we find that the Löwdin transformation amplifies non-two-center effects, as may be seen from the expansion in $\Delta \mathrm{O} \equiv \mathbf{O}-\mathbf{1}$.

$$
\mathbf{H}^{\text {or }}=\mathbf{H}-\frac{1}{2}(\Delta \mathbf{O} \cdot \mathbf{H}+\mathbf{H} \cdot \Delta \mathbf{O})+\cdots
$$

Terms which are of first and higher order in $\Delta \mathrm{O}$ involve products of the geometric matrices which, due to the incompleteness of the $\mathrm{g}_{\mu}\left(\ell, \ell^{\prime}, \hat{\mathbf{R}}\right) \equiv\left[g_{\mu}\left(\ell m, \ell^{\prime} m^{\prime}, \hat{\mathbf{R}}\right)\right]$, add non-two-center contributions to the $\mathbf{H}^{\text {or }}$ matrix elements. We consistently find degraded agreement between the orthogonal Hamiltonian matrix elements, $\left\langle 0 \ell m\left|H^{\mathrm{or}}\right| \mathbf{R} \ell m^{\prime}\right\rangle$ and their tight binding reconstructions, as well as between the corresponding band structures, in comparison to the nonorthogonal case. These non-two-center effects diminish for more localized bases. In order to focus solely on the effects of orthogonalization in this section, we use a basis which is sufficiently localized that the minimal basis, nonorthogonal tight binding, and orthogonal tight binding band structures are all three essentially identical. This agreement comes at the cost, however, of poor representation of the accurate multiple- $\kappa$ band structure.

Figure 3 shows calculations of nonorthogonal, $t$, and orthogonal, $t^{\text {or }}$, hopping parameters for the $\mathrm{fcc}$ and $\mathrm{cd}$ phases of $\mathrm{Si}$, with appropriate sign changes as required to plot positive numbers. As in Fig. 2, the calculated parameters appear in triples corresponding to $V_{0}$ and $(1 \pm 0.2) V_{0}$. They are connected by lines (solid, fcc; dashed, cd) for visual convenience, and the nonorthogonal parameters have been multiplied by three (" $3 t$ ") to separate the data. The minimal basis chosen corresponded to a single augmented spherical Hankel function $\left(\kappa^{2}=0.5 \mathrm{Ry}\right)$ for each atomic orbital. In addition, we used smaller, fixed muffin-tin radii $\left(R_{\mathrm{MT}}=1.7 \mathrm{Bohr}\right)$ for all atoms in order to switch sooner from the numerically generated radial wave functions to the faster decaying Hankel function tails. For comparison, touching muffin-tins in $V / V_{0}=0.8 \mathrm{~cd}$ Si would have $R_{\mathrm{MT}}=2.05 \mathrm{Bohr}$. The present nonorthogonal-case overlap parameters, $s_{\ell \ell^{\prime} \mu}(d)$, are already so consistent among the two structures and three volumes, that there was no need to perform unitary transformations to guarantee this transferability. The $s p$ subset of these $s_{\ell \ell^{\prime} \mu}(d)$ are smaller by factors of 1.6-3.8 at the near-neighbor distance, and by factors of 2.2-6.9 at the third neighbor distance, in comparison with our optimum linked-basis parameters used in Fig. 2, which provided good agreement with the multiple- $\kappa$ bands.

The first feature to note in Fig. 3 is that the nonorthogonal $t$ 's are in relatively close agreement between the two structures and for all three volumes. While the fcc nearncighbor $t_{\ell \ell^{\prime} \sigma}$ 's appear to be slightly lower than their cd counterparts, recall that a rigid- 


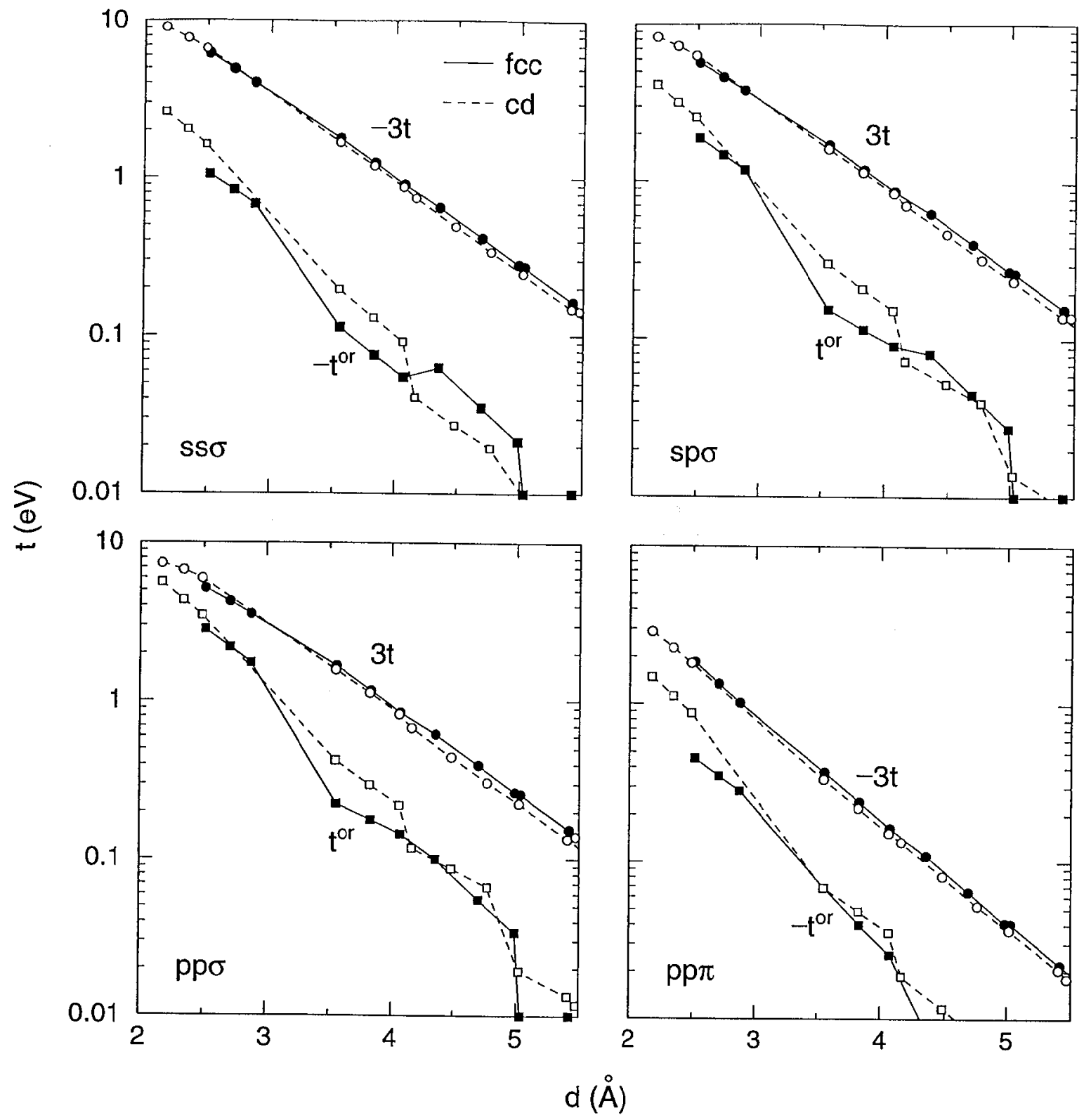

Figure 3: Nonorthogonal hopping parameters multiplied by three (circles, $3 t$ ) and orthogonal hopping parameters (squares, $t^{\text {or }}$ ) for fcc (filled symbols, solid lines) and cd Si (open symbols, dashed lines). Some fcc $t^{\text {or }}$ for $d>5 \AA$ have changed sign, which is indicated by plotting these points on the bottom axes of the three $\ell \ell^{\prime} \sigma$ panels.

band shift could have achieved the same result for Fig. 2. More significant is the slight rise here with increasing $d$ of the fcc $t$ 's relative to the cd values, a trend which was fully evident in Fig. 2. These are 10-20\% effects, whereas the orthogonal basis hopping parameters, $t^{\text {or }}$, in Fig. 3 are clearly much less transferable. The $V / V_{0}=1.2 \mathrm{~cd}$ and $V / V_{0}=0.8 \mathrm{fcc}$ nearneighbor distances are nearly the same, yet the $\mathrm{cd} t^{\text {or }}$ magnitudes here are larger by factors of 1.2-1.9 than the corresponding fcc values. Similarly, the second neighbor distances are fairly close, and here the cd magnitudes are a factor of $\sim 1.7$ larger than the fcc values.

We have analyzed the effect of orthogonalization using the first order terms in $\Delta \mathrm{O}$ shown in Eq.(14). To verify that this would reasonable, we first numerically calculated the 
$t^{\text {or }}(d)$ over the range $0 \leq \eta \leq 1$, corresponding to the Löwdin orthogonalization of $\mathbf{H}$ using a scaled overlap, $\mathbf{1}+\eta(\mathbf{O}-\mathbf{1})$. The usual nonorthogonal, $t$, and orthogonal, $t^{\text {or }}$, parameters correspond to the $\eta=0$ and 1 limits, respectively. We generally found that the first order corrections, $\left.\left(d t^{\circ r} / d \eta\right)\right|_{\eta=0}$, to the $t^{\prime}$ s were of the same sign although significantly larger (factors of 2 or so) than the exact corrections, $t^{\text {or }}-t$. Given that the signs of the first order corrections tend to be right, we then used Eq.(14) to obtain analytic expressions for these terms for further investigation [20]. In the absence of crystal field, Eq.(14) implies

$$
\begin{aligned}
t_{\ell \ell^{\prime} \mu}^{\mathrm{or}}(R)= & t_{\ell \ell^{\prime} \mu}(R)-\frac{1}{2}\left(\varepsilon_{\ell}+\varepsilon_{\ell^{\prime}}\right) s_{\ell \ell^{\prime} \mu}(R) \\
& -\frac{1}{2\left(2-\delta_{\mu \sigma}\right)} \sum_{\mathbf{R}^{\prime}}^{\neq 0, \mathbf{R}} \sum_{\lambda, \mu_{1}, \mu_{2}} \mathbf{g}_{\mu}\left(\ell, \ell^{\prime}, \hat{\mathbf{R}}\right) \cdot \mathbf{g}_{\mu_{1}}\left(\ell, \lambda, \hat{\mathbf{R}^{\prime}}\right) \times \mathbf{g}_{\mu_{2}}\left(\lambda, \ell^{\prime}, \widehat{\left.\mathbf{R}-\mathbf{R}^{\prime}\right)}\right. \\
& \times\left[t_{\ell \lambda \mu_{1}}\left(R^{\prime}\right) s_{\lambda \ell^{\prime} \mu_{2}}\left(\left|\mathbf{R}-\mathbf{R}^{\prime}\right|\right)+s_{\ell \lambda \mu_{1}}\left(R^{\prime}\right) t_{\lambda \ell^{\prime} \mu_{2}}\left(\left|\mathbf{R}-\mathbf{R}^{\prime}\right|\right)\right]+\cdots
\end{aligned}
$$

Here, $\mathbf{g}_{\mu}\left(\ell, \ell^{\prime}, \hat{\mathbf{R}}\right) \equiv\left[g_{\mu}\left(\ell m, \ell^{\prime} m^{\prime}, \hat{\mathbf{R}}\right)\right]$, the $\times$ signifies regular matrix multiplication, and the dot product, $\sum_{m, m^{\prime}}$, so that the triple product of gcometric matrices is a scalar. The bond atoms at 0 and $\mathbf{R}$ together with a neighboring atom at $\mathbf{R}^{\prime}$ define triangles with angles $\theta_{1}$ and $\theta_{2}$ adjacent to the bond, and $\theta_{3}=\pi-\theta_{1}-\theta_{2}$ opposite to the bond. 'I'he triple product of geometric matrices depends only on these angles, and for the simplest $t_{s s \sigma}^{\text {or }}$ case is 1 and $-\cos \theta_{3}$ for the $\lambda=s$ and $p$ contributions, respectively. Both make the $t_{s s \sigma}^{\text {or }}$ more positive, i.e., reduce its magnitude, if $0_{3}<90^{\circ}$. More generally we find for an $s p$ basis that most of the contributions tend to reduce the magnitudes of the $t_{\ell \ell^{\prime} \mu}^{\text {or }}$ if all angles are acute, $0<\theta_{1}, \theta_{2}, \theta_{3}<90^{\circ}$. It is a characteristic of the more close packed phases that not only are there more neighbors closer to the shorter bond axes, implying larger contributions to Eq.(15), but the associated angles also tend to all be acute so that the $t^{\text {or }}$ are generally reduced in magnilude. For more open packed structures, on the other hand, there are fewer nearby neighbors, implying reduced impact of the orthogonalization, and for the cd structure, the smallest triangles have an obtuse angle, so there is greater possibility for cancellation amongst the terms in Eq.(15). These geometric considerations appear consistent with the results in Fig. 3.

\section{SUMMARY}

Current interest in tight-binding representations is driven by the need for fast yet still accurate calculation of interatomic forces for use in molecular statics and dynamics calculations. Given the success of local-density functional methods in the calculation of total energies and forces, it makes sense to analyze these methods from a tight-binding perspective both to obtain $a b$ initio values of the parameters as well as to gain insight into the validity of approximations and assumptions customarily made in tight-binding treatments.

Two fundamental approximations arc madc in typical tight binding represcntation of one-electron matrices or band structures: use of a minimal basis and the two-center approximation. Although there are some instances where the former approximation could be improved, we find on the whole that the use of a minimal basis is quite satisfactory, as seen both here for fcc Ce and in earlier work [11] for Si/B phases. The two center expansion is by far the more serious approximation. Whilc wc find this approximation to be quite good for relatively localized bases, we consistently find those minimal bases which 
most accurately reproduce our best multiple- $\kappa$ bands to be also accompanied by noticeable non-two-center effects. This is particularly evident here in $d$-like states at the bottom of the Ce conduction band, as well as in the conduction bands of four-fold coordinated Si/B structures [11]. Even the $4 \int$ states in Ce which should be relatively localized are not immune. In this regard, we have noted that the two-center approximation is likely to become worse with increasing $\ell$.

We have also investigated the transferability of our ab initio calculated Slater-Koster parameters across a range of coordinations for the case of $\mathrm{Si}$, in both nonorthogonal and orthogonal representalions. In agreement with generally held expectations, we find the nonorthogonal parameters to have far better transferability. But even for the nonorthogonal parameters there is residual coordination dependence which probably originates from the three-center terms. It is interesting that three-center terms may well be the source of both of the problems discussed in this work. If one imagines separating each such term into a component orthogonal to and another spanned by the space of two-cenler geometric matrices, then the former contribute to inadequacies of the two-center approximation, while the latter likely contribute to environmental dependence, i.e., non transferability, of the two-center Slater-Koster parameters. The use of environmentally dependent fitting procedures [9] for the hopping parameter addresses the second but not the first aspect of this compound three-center problem.

It should be acknowledged that tight-binding total-energy representations do not require the one-electron matrix elements themselves, which determine both band structure and eigenvectors, but only the former. The less demanding representational problem of the band structure alone provides more freedom for effective two-center parameters to incorporate what are truly non-two-center effects. Given the fundamental goal of accurate total energies and forces, it may well be that such effective parameters are entirely adequate, and for that matter, that accurate representation of the ab initio local density functional bands is not critical. On the other hand, local density functional theory is itself a paradigm for accurate transferable forces, and clear suggestions for improved tight-binding representations arisc from its cxamination. One is to go beyond the two-center approximation by augmenting the current space of two-center geometric functions. A second point is to affirm the use [7] of environmentally dependent site energies as a more flexible format for incorporating the interatomic potential terms ususally incorporated in tight-binding total-energy representations.

\section{ACKNOWLEDGMENTS}

This work was performed under the auspices of the U.S. Department of Energy by Lawrence Livermore National Laboratory under Contract No. W-7405-Eng-48. We are grateful to M. van Schilfgaarde for providing a new version of the FP-LMTO codes which was used in carrying out the linked basis calculations described here.

\section{REFERENCES}

1. R. Car and M. Parrinello, Phys. Rev. Lett. 55, 2471 (1985).

2. G. Galli, A. Selloni, and R. Car, Chimia 47, 313 (1993).

3. L. Colombo, Annual Reviews of Computational Physics IV, edited by D. Stauffer (World Scientific, 1996), p. 147. 
4. L. Goodwin, A. J. Skinner, and D. G. Pettifor, Europhys. Lett. 9, 701 (1989).

5. S. Sawada, Vacuum 41, 612 (1990); M. Kohyama, J. Phys.:Condens. Matter 3, 2193 (1991).

6. I. Kwon, R. Biswas, C. Z. Wang, K. M. Ho, and C. M. Soukoulis, Phys. Rev. B 49, 7242 (1994).

7. R. E. Cohen, M. J. Mehl, and D. A. Papaconstantopoulos, Phys. Rev. B 50, 14694 (1994); M. J. Mehl, D. A Papaconstantopoulos, R. E. Cohen, and M. M. Sigalas, in Alloy Modeling and Design, edited by G. Malcolm Stocks (The Minerals, Metals, and Materials Society, Warrendale, Pennsylvania, 1994), p. 25; M. J. Mehl, D. A. Papaconstantopoulos, and R. E. Cohen, Int. J. Thermophys. 16, 503 (1995).

8. J. L. Mercer, Jr. and M. Y. Chou, Phys. Rev. B 49, 8506 (1991)

9. M. S. Tang, C. Z. Wang, C. T. Chan, and K. M. Ho, Phys. Rev. B 53, 979 (1996).

10. T. J. Lenosky, J. D. Kress, I. Kwon, A. F. Voter, B. Edwards, D. F. Richards, S. Yang, and J. B. Adams, Phys. Rev. B 55, 1528 (1997).

11. A. K. McMahan and J. E. Klepeis, Phys. Rev. B 56, 12250 (1997).

12. M. Methfessel, Phys. Rev. B 38, 1537 (1988); M. Methfessel, C. O. Rodriguez, and O. K. Andersen, Phys. Rev. B 40, 2009 (1989).

13. J. C. Slater and G. F. Koster, Phys. Rev. 94, 1498 (1954).

14. W. A. Harrison, Electronic Structure and the Properties of Solids, (Dover, New York, 1989), p. 479.

15. Rigid band shifts were not taken into account in the discussion of Table IV in [11], and would significantly improve the transferability of the "Table II $t$ 's" discussed there.

16. O. K. Andersen, O. Jepsen, and D. Glötzel, in Highlights of Condensed Matter Theory, cditcd by F. Bassani, F. Fumi, and M. P. Tosi (North-Holland, Amsterdam, 1985, p. 59; H. L. Skriver, The LMTO Method (Springer, Berlin, 1984).

17. J. C. Duthie and D. G. Pettifor, Phys. Rev. Lett. 38, 564 (1977).

18. For references and an application of the force theorem, see, e.g., A. K. McMahan and J. A. Moriarty, Phys. Rev. B 27, 3235 (1983).

19. P. O. Löwdin, J. Chem. Phys. 19, 1396 (1951).

20. The Löwdin expansion has also been discussed by J. Dorantes-Dávila, A. Vega, and G. M. Pastor, Phys. Rev. B 47, 12995 (1993). 


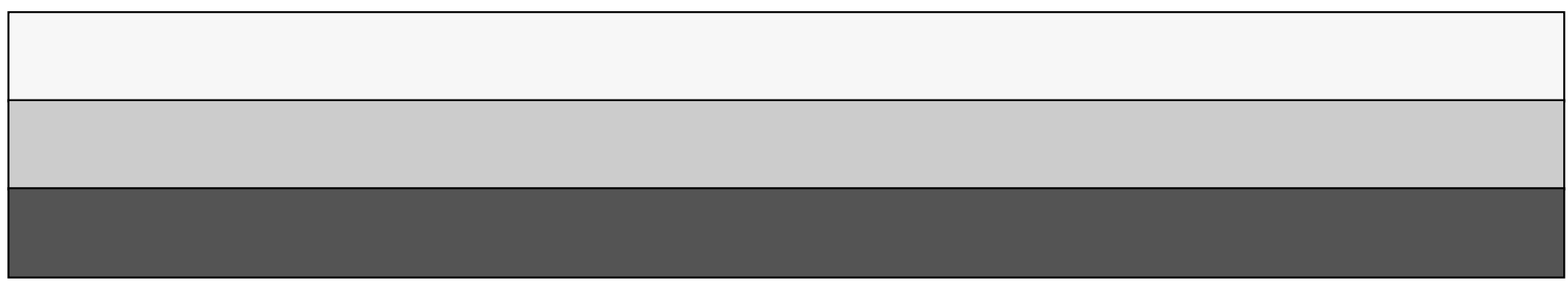

